\title{
Methodologische Einführung zur 8. und 9. Auflage
}

Uwe Quasthoff

\author{
1. Übersicht \\ 2. Das Vorgehen zur Neubearbeitung \\ 2.1. Hintergrund: Das Projekt Deutscher Wortschatz \\ 2.2. Entscheidung für den korpusbasierten Ansatz \\ 2.3. Erzeugen einer elektronischen Version \\ 2.4. Auswahlkriterien für die Aufnahme von Wörtern und Wortgruppen \\ 2.5. Herausnehmen niederfrequenter neuer Wörter und Wortgruppen aus \\ den Wörterbuchartikeln \\ 2.6. Einordnung neuer Wörter und Wortgruppen in sachgruppenbezogene \\ Wörterbuchartikel \\ 2.7. Verweise auf andere sachgruppenbezogene Wörterbuchartikel \\ 2.8. Inhaltliche Änderungen \\ 3. Die sachgruppenbezogenen Wörterbuchartikel und ihre Anordnung \\ 3.1. Grobgliederung in Sachgruppen \\ 3.2. Feingliederung der sachgruppenbezogenen Wörterbuchartikel
}

\section{1. Übersicht}

Diese methodologische Einführung wendet sich an Leser mit Interesse an Wörterbuchherstellung und Wörterbuchforschung. Ausführlich beschrieben wird das für die Neubearbeitung zu Grunde gelegte Material und die vorgenommenen Arbeitsschritte; nicht genauer eingegangen werden kann allerdings auf die im Zusammenhang mit Kookkurrenzen verwendeten statistischen Verfahren.

Das Ziel, den größeren Teil des Wortschatzes der Alltagssprache in seinen semantischen Zusammenhängen darzustellen, verlangt zunächst nach einem möglichst universellen Auswahlkriterium für die aufzunehmenden Wörter. Die Wahl fiel auf einen so genannten korpusbasierten Ansatz: Aus einer sehr großen Textmenge wurden mittels Häufigkeitsanalyse Wörter für die Neuaufnahme ausgewählt. Weiterhin konnte in vielen Fällen eine oder mehrere Sachgruppen für die Zuordnung eines so gefundenen neuen Wortes automatisch vorgeschlagen werden. Die abschließende Entscheidung über die Aufnahme und die Einordnung wurde vom Bearbeiter vorgenommen. 
Der korpusbasierte Ansatz sorgt dafür, dass große Bereiche der Alltagssprache abgedeckt werden. Während Fachsprachen (z.B. die Bereiche Computer, Medizin oder Sport) in den alltäglichen Sprachbereich hineinreichen, werden regionales Wortgut sowie Gruppen- und Sondersprachen nur eingeschränkt berücksichtigt. Dies ist vor allem eine Folge des korpusbasierten Ansatzes. Das Auswahlverfahren wirkt sich allerdings positiv auf die „Lesbarkeit“ des Wörterbuches aus. Die einzelnen sachgruppenbezogenen Wörterbuchartikel und die darin enthaltenen kleineren semantischen Gruppen sind wesentlich homogener geworden in dem Sinne, dass die aufgeführten Wörter und Wortgruppen ${ }^{1}$ weniger sprachliche Ausdrücke enthalten, die kaum allgemein bekannt sind. Unbekanntes Wortmaterial wird vom Benutzer auch nicht angenommen, da es bei der Wörterbuchbenutzung des Deutschen Wortschatz nach Sachgruppen in erster Linie darum geht, das Finden von Wörtern oder Wortgruppen, die der Benutzer bereits kennt, an die er sich aber nicht erinnern kann, zu unterstützen. Werden Wörter gefunden, die der Benutzer nicht kennt, wären erklärende und unterscheidende Angaben erforderlich, die in diesem Wörterbuch nicht vorgesehen sind.

Da die lexikographische Pflege des vorliegenden Wörterbuchs vor der achten Auflage über mehr als 4 Jahrzehnte ruhte, sollte man nicht erwarten, dass die vorliegende Bearbeitung alle Wünsche an die Auswahl der Wörter und Wortgruppen und deren Einordnung erfüllt. Das Sachgruppensystem ist offen für Veränderungen: Es ist variabel und erweiterungsfähig; auch verträgt es Umstellungen, ohne dass die Benutzbarkeit leidet.

\section{Das Vorgehen zur Neubearbeitung}

\subsection{Hintergrund: Das Projekt Deutscher Wortschatz}

Seit März 1998 sind die Daten des Projekts Deutscher Wortschatz unter wortschatz.unileipzig.de im Internet verfügbar. Die vorhandenen Daten gliedern sich in zwei Teile, das Wortschatz-Korpus und das Wortschatz-Lexikon. Diese Datensammlungen entstanden an der Abteilung Automatische Sprachverarbeitung am Institut für Informatik der Universität Leipzig zunächst aus dem Grund, dass Mitte der Neunziger Jahre praktisch keine frei verfügbaren elektronischen Wörterbücher für die deutsche Sprache existierten. Dabei war es nicht primär das Ziel, ein elektronisches Wörterbuch zu schaffen, welches mit den gedruckten Wörterbüchern in Konkurrenz tritt. Vielmehr verlangten verschiedene Fragestellungen aus dem Komplex Automatisches Textverstehen nach sehr

1 Der Ausdruck Wortgruppen wird im Folgenden verwendet für alle Arten von Syntagmen, seien sie nicht idiomatisch, teil- oder vollidiomatisch. 
viel mehr Informationen über Wörter und Wortgruppen als zur Verfügung standen. Diese Fragestellungen betreffen Sachgebietszuordnungen für Wörter und Texte, Erkennen von Fachausdrücken und Eigennamen sowie das Erkennen von Zusammenhängen zwischen verschiedenen Wörtern. Spezielles Interesse gilt seltenen (d.h. niederfrequenten) Wörtern, da diese in der Regel in sehr speziellen Situationen (z.B. einem speziellen fachsprachlichen Kontext) gebraucht werden und somit einen großen Informationsgehalt besitzen. Um solche niederfrequenten Wörter zu finden, wurden sehr viele Texte gesammelt.

Dieses Wortschatz-Korpus wird ständig erweitert und dient dazu, ein aktuelles Vollformenwörterbuch zu erzeugen. Aus dem Textkorpus werden die auftretenden Wortformen extrahiert und durch ausgewählte Wortgruppen ergänzt. Dies bildet die Lemmaliste des Wortschatz-Lexikons. Zu den Wortformen werden verschiedenste Angaben gesammelt oder mit überwiegend automatischen Mitteln erzeugt. Ergebnis ist ein Wörterbuch mit Angaben zur Grammatik, zu Sachgebieten und zur Verwendung. Das Korpus liefert außerdem Belegstellen für die Wörter und Wortgruppen. Von besonderem Interesse sind hier so genannte Kookkurrenzen, dies sind Paare von Wörtern, die statistisch auffällig gemeinsam innerhalb eines Satzes vorkommen. Für jedes hinreichend häufige Wort (z. B. Kaffee) lässt sich nun eine so genannte Kookkurrenzmenge bilden, dies ist die Menge der mit Kaffee am auffälligsten gemeinsam auftretenden Wörter. Diese Auffälligkeit wird als Abweichung von der statistischen Unabhängigkeit gemessen und liefert einen Zahlenwert, nach dem sich diese Kookkurrenzmenge ordnen lässt. Die stärksten Kookkurrenzen für Kaffee sind beispielsweise Tasse, Tee, trinken, Kuchen, trinkt, Tassen, Zucker, getrunken, Kakao, ... Mit einfachen Stimulus-ResponseExperimenten wurde gezeigt, dass solche Kookkurrenzen sehr gut mit menschlichen Assoziationen übereinstimmen: Wörter, die einem Menschen nach Nennung eines Stimulus-Wortes (z.B. Kaffee) schnell einfallen (wie z.B. Tee, Tasse, trinken), sind auch die stärksten Kookkurrenzen des Stimulus-Wortes. ${ }^{2}$

Dass sich diese menschlichen Assoziationen mit statistischen Mitteln aus einem großen Textkorpus ermitteln lassen, ist nicht verwunderlich. Beim Verfassen der Texte fließen solche Assoziationen des Autors bewusst oder unbewusst in seine Texte ein. Liegt genügend viel Text vor, lassen sich diese Zusammenhänge als Abweichungen von der statistischen Unabhängig berechnen.

Deshalb wird beim Betrachten solcher Kookkurrenzmengen sofort klar, dass sie sehr gutes Ausgangsmaterial für die Überarbeitung des Dornseiff darstellen.

2 Siehe z. B. Reinhard Rapp (1996): Die Berechnung von Assoziationen: ein korpuslinguistischer Ansatz. Hildesheim, Zürich, New York: Olms. (Reihe: Sprache und Computer, herausgegeben von P. Hellwig und J. Krause, Bd. 16) 


\subsection{Entscheidung für den korpusbasierten Ansatz}

Ende 1998 erreichte den Bearbeiter die Anfrage des Verlages, ob es möglich wäre, mit Hilfe der Daten des Projekts Deutscher Wortschatz die längst überfällige Neubearbeitung des Dornseiff vorzunehmen. Da die entstehenden Daten auch für die weiteren Forschungsvorhaben im Bereich Automatisches Textverstehen von Nutzen sein würden, schien die Aufgabenstellung interessant. Schnell zeigte sich, dass die Datengrundlage im Wortschatz-Lexikon ausreichend war, da für die beispielhaft getesteten Wörter, die im Dornseiff verbleiben sollten, jeweils mehr als zehn Belegstellen gefunden wurden. Mit den Kookkurrenzen schien auch eine Möglichkeit gegeben, die richtige Stelle für neu einzuordnende Wörter zu finden. Mit so vielseitiger maschineller Unterstützung erschien das Vorhaben in einem vernünftigen Zeitrahmen durchführbar.

Natürlich war schon damals klar, dass mit den maschinellen Methoden immer nur Vorschläge für die Aufnahme einzelner Wörter erzeugt werden können. Danach ist die menschliche Kompetenz gefordert, die Vorschläge der Maschine anzunehmen oder zu verwerfen. Der Fortschritt besteht darin, dass diese Entscheidung viel schneller zu fällen ist als die traditionelle intellektuelle Suche nach der richtigen Stelle, an der ein Wort einzuordnen ist. Die Zeitersparnis gegenüber der traditionellen Methode beträgt hier rund 90\%. Dies wurde andererseits auch wieder schmerzhaft bewusst in Bereichen, in denen die automatischen Verfahren beispielsweise deshalb nicht funktionieren konnten, weil es entsprechende Sachgruppen bisher noch nicht oder nur rudimentär gab. Hier fehlte das Gerüst, das den automatischen Verfahren Halt bei der Einordnung gibt.

Da weiterhin klar war, dass ein einzelner Bearbeiter nicht die fachliche Kompetenz in allen Fachgebieten haben konnte, wurden für ausgewählte Bereiche Fachgutachter hinzugezogen.

Unter diesen Voraussetzungen kam im Frühjahr 1999 die Entscheidung zustande, eine korpusbasierte Neubearbeitung des Dornseiff in Angriff zu nehmen.

\subsection{Erzeugen einer elektronischen Version}

Der erste Schritt bestand in einer Sichtung und Aufbereitung der vorhandenen Materialien. Vom Verlag wurden die für den Druck der siebten Auflage benutzten Filme zur Verfügung gestellt. Die sachgruppenbezogenen Wörterbuchartikel wurden zunächst eingescannt. Diese daraufhin als Bilddaten vorliegenden Textseiten wurden mittels optischer Zeichenerkennung (OCR) in ein Textverarbeitungssystem übernommen. Nach den notwendigen manuellen Korrekturen lag die siebte Auflage als Textdatei vor.

Bereits in der siebten Auflage waren Wörter und Wortgruppen zu semantischen Gruppen zusammengefasst und nach Wortarten sortiert; diese Einteilungen mussten in der elektronischen Form nachvollzogen werden. Zusätzlich wurden die Eigenschaften 
der semantischen Gruppe (wie Wortart und Wörter oder Wortgruppen) notiert, um diese Informationen später nutzen zu können. Dieses Textdokument diente als Ausgangspunkt für die weitere Bearbeitung der Wörterbuchartikel und soll hier als Manuskript bezeichnet werden.

\subsection{Auswahlkriterien für die Aufnahme von Wörtern und Wortgruppen}

Die Qualität einer korpusbasierten Auswahl hängt bekanntlich vom zugrundeliegenden Korpus ab. Im Idealfall sollte solch ein Korpus sehr groß sein, um zufällige statistische Fluktuationen auszublenden. Es sollte weiterhin möglichst ausgewogen sein, was den Anteil aus verschiedenen Textsorten und Varietäten betrifft. Wir gehen von der Vorstellung aus, dass unter diesen Bedingungen die Häufigkeit eines Wortes oder einer Wortgruppe in unserem Textkorpus seine Bekanntheit unter den Sprechern ausdrückt und damit ein sinnvolles Kriterium für die Aufnahme in das Wörterbuch ist. Aus mehreren Gründen sind hier allerdings Relativierungen notwendig.

Das größte Problem bildet die gesprochene Sprache: Sie ist einerseits sehr reich an idiomatisch geprägten Wendungen, andererseits in großen maschinenlesbaren Sammlungen stark unterrepräsentiert. Als ausgleichende Maßnahme wurde die Schwelle für die Aufnahme von Wortgruppen im Vergleich zu Wörtern deutlich gesenkt.

Die zweite Problemgruppe umfasst veraltende Wörter. Diese sind häufig bekannt, aber ihre Verwendung in aktuellen Texten ist seltener als ihre Bekanntheit erwarten ließe. Hier wurde davon ausgegangen, dass die zu berücksichtigenden veraltenden Wörter in der siebten Auflage vorhanden sind. Die Schwelle für das Herausnehmen vorhandener Wörter wurde nun niedriger gesetzt als die Schwelle für die Aufnahme neuer Wörter, so dass vorhandene Wörter auch mit niedrigerer Häufigkeit im Wörterbuch belassen wurden, während neue Wörter mit vergleichbarer Häufigkeit noch nicht aufgenommen wurden.

Nicht so problematisch ist hingegen die Tatsache, dass Polysemie bei der Häufigkeitsanalyse zunächst unberücksichtigt bleibt. Bei der späteren Einordnung mit Hilfe von Kookkurrenzen wird dies automatisch nachgeholt, weil nur für hochfrequente Bedeutungen Kookkurrenzen gefunden werden und niederfrequente Bedeutungen damit automatisch unberücksichtigt bleiben.

Bei der achten Auflage wurde folgendes Auswahlverfahren angewandt: Als Korpus wurde das oben beschriebene Wortschatz-Korpus der Abteilung Automatische Sprachverarbeitung am Institut für Informatik der Universität Leipzig (www.wortschatz.unileipzig. de) verwendet. Zum Zeitpunkt der Auswertung bestand die Textsammlung aus rund 230 Millionen laufenden Wörtern, die entspricht etwa 800.000 eng bedruckten DIN-A4-Seiten. Die Sammlung besteht im Wesentlichen aus Zeitungstexten aus den Jahren 1987-2001, enthält aber auch Literatur und populärwissenschaftliche Texte. 
Wörter mit einer absoluten Häufigkeit von mindestens 20 Vorkommen wurden zur Aufnahme vorgeschlagen. Um mit veraltenden Wörtern toleranter umzugehen, wurden Wörter aus der siebten Auflage nur dann zum Herausnehmen vorgeschlagen, wenn sie eine Häufigkeit von höchstens 10 Vorkommen hatten. Bei Wortgruppen wurde die Toleranzschwelle weiter gesenkt, hier wurden nur solche zurückgewiesen, für die es überhaupt keine Belegstellen im Korpus gab.

Bis zum Jahr 2003 wurde das Wortschatz-Korpus auf rund 500 Millionen laufende Wörter erweitert. Eine Vergrößerung des Umfangs erscheint sinnvoll, um für spätere Auflagen genauere Entscheidungen treffen zu können. Die folgenden Beispiele zeigen herausgenommene und beibehaltene Wortgruppen aus 10.44 Eitelkeit. Die in Klammern angegebenen absoluten Häufigkeiten beziehen sich auf zur Auswahl benutzte Wortschatz-Korpus (erste Zahl) sowie auf die Internet-Suchmaschine Google (zweite Zahl). Die von Google indexierte deutschsprachige Textmenge war im Jahr 2003 sehr grob geschätzt 100 mal größer als das zur Auswahl benutzte Wortschatz-Korpus.

Aus 10.44 Eitelkeit wurden folgende Wortgruppen herausgenommen: den dicken Wilhelm markieren $(\mathrm{o} / 7) \cdot$ er hat einen Gickel $(\mathrm{o} / \mathrm{o}) \cdot(\mathrm{geht})^{3}$ mit großen Hunden pissen $(\mathrm{o} / 7) \cdot$ große Bogen spucken $(\mathrm{o} / 2) \cdot$ sich mausig machen $(\mathrm{o} / 36) \cdot$ nach Auszeichnungen haschen $(\mathrm{o} / \mathrm{o}) \cdot \ldots$

In 10.44 Eitelkeit bleiben erhalten: (sich) für etwas Besonderes halten $(1 / 85) \cdot$ sich für ein Genie halten $(1 / 36) \cdot($ sich $)$ in die Brust werfen (12/142) • (sich) in den Vordergrund drängen $(17 / 142) \cdot$ das Maul aufreißen $(10 / 541) \cdot$ sich mit fremden Federn schmücken $(10 / 1310) \cdot \ldots$

Die Zahlen zeigen einerseits, dass beide Korpora keine widersprüchlichen Zahlenwerte liefern, aber auch, dass ein noch größeres Korpus eine bessere Entscheidungsgrundlage liefert.

Während es relativ einfach ist, neu aufzunehmende Wörter vorzuschlagen, ist die analoge Aufgabe für Wortgruppen schwieriger, da Kandidaten für aufzunehmende Wortgruppen erst einmal vorliegen müssen, um dann mit Hilfe der für sie bestimmten Anzahl über die Aufnahme zu entscheiden. Hier konnte aber auf bereits im WortschatzLexikon vorliegende Wortgruppen zurückgegriffen werden.

Als Konsequenz des korpusbasierten Auswahlverfahrens hat sich beim Übergang von der siebten zur achten Auflage die Zusammensetzung vieler sachgruppenbezogener Wörterbuchartikel (im Folgenden auch kurz Wörterbuchartikel genannt) geändert. Viel seltenes Wortmaterial wurde herausgenommen; aber in den meisten Fällen war dieses für den Benutzer als veraltetes oder regionales Wortgut ohnehin nicht produktiv nutzbar. Die neuen Wörterbuchartikel zeichnen sich durch eine größere Homogenität in der Auswahl und bessere Benutzbarkeit aus: Die jeweils angeführten Wörter und

3 Die in runde Klammern gesetzten Teile der Wortgruppen wurden bei der Suche mit Google nicht berücksichtigt. 
Wortgruppen sollten sich neben ihrer inhaltlichen Nähe auch in Bekanntheit und Anwendbarkeit ähneln.

Für die neunte Auflage wurde die semantische Struktur des Dornseiff weitestgehend unverändert gelassen. Der Schwerpunkt der Neubearbeitung bestand in der Aufnahme in der Erweiterung der Menge der verzeichneten Wörter. Grundlage bildete wieder das Wortschatz-Korpus, welches bis 2017 auf reichlich fünf Milliarden laufende Wörter angewachsen war.

Um die quantitativen Auswirkungen dieses Auswahlverfahrens zu erkennen, sollen die Einträge in den Wörterbuchartikeln gezählt werden. ${ }^{4}$ Unter einem Eintrag verstehen wir hier Wörter oder Wortgruppen, die in den Wörterbuchartikeln genannt werden und jeweils durch Mittenpunkte oder quadratische Trennzeichen getrennt sind. Beim Vergleich der Zahl der Einträge lassen sich sowohl die Gesamtzahl der Einträge wie auch die Anzahl der verschiedenen Einträge auswerten. Die Anzahl der verschiedenen Einträge entspricht dabei der Anzahl der Registereinträge im vollständigen alphabetischen Zugriffsregister. Die Zahl der entfallenen Einträge und die daraus berechnete Anzahl der neu aufgenommenen Einträge konnte nur geschätzt werden, da in einigen Fällen Wortgruppen jetzt in einer anderen Form (z. B. neu: über die Kraft gehen statt alt: geht über die Kraft) aufgenommen sind und solche Änderungen nicht gezählt werden sollen.

\begin{tabular}{lcccccc} 
& 7. Aufl. & \multicolumn{3}{c}{ 8. Aufl. } & \multicolumn{2}{c}{ 9. Aufl. } \\
& gesamt & gesamt & entfallen & neu & gesamt & neu \\
Gesamtzahl der Einträge & 91746 & 89582 & 29500 & 27336 & 107476 & 20763 \\
Anzahl verschiedener Einträge & 56450 & 53737 & 24500 & 21787 & 68160 & 16045
\end{tabular}

\subsection{Herausnehmen niederfrequenter neuer Wörter und Wortgruppen aus den Wörter- buchartikeln}

Der erste Bearbeitungsschritt des Manuskripts (dies war zu diesem Zeitpunkt noch die fast unveränderte siebte Auflage) bestand im Herausnehmen der als zu niederfrequent betrachteten Wörter und Wortgruppen. Dies erfolgte vollautomatisch durch Ausnutzung der Häufigkeitsangaben aus dem Wortschatz-Lexikon und den oben angegebenen Mindesthäufigkeiten für Wörter und Wortgruppen. Die auf diese Weise herausgenommenen Wörter und Wortgruppen stehen für weitere Untersuchungen zur Verfügung und finden möglicherweise einen Platz auf der CD-ROM-Version zu späteren Auflagen dieses Wörterbuches. ${ }^{5}$

4 Bei den Größenvergleichen werden die beiden Ausnahme-Sachgruppen 2.2 Pflanzenarten und 2.8 (ehemals 2.9) Tierarten der siebten Auflage nicht berücksichtigt, da beide in Sachgruppen wie in Abschnitt 2.8 beschrieben sowohl in der Länge wie im Inhalt völlig aus dem Rahmen fielen.

5 Wer den Dornseiff verwenden möchte, um archaisierende Texte zu verfassen, wird auf die siebte Auflage verwiesen. 


\subsection{Einordnung neuer Wörter und Wortgruppen in sachgruppenbezogene Wörter- buchartikel}

Der nächste Schritt nach der Auswahl der neu aufzunehmenden Wörter und Wortgruppen ist deren Zuordnung zu Sachgruppen sowie die Einordnung in die angemessene semantische Gruppe innerhalb der dazugehörigen sachgruppenbezogenen Wörterbuchartikel ${ }^{6}$. Außerdem ist zu entscheiden, ob ein Wort oder eine Wortgruppe an nur einer oder an mehreren Stellen eingeordnet werden soll. Diese Entscheidung hängt nicht nur davon ab, ob das entsprechende Wort oder die Wortgruppe mehrere Bedeutungen hat (z. B. Band bei 4.33 Verbinden, 12.58 Buch oder 14.14 Ensemble), da beispielsweise auch Wörter mit nur einer Bedeutung in mehreren Zusammenhängen eingeordnet sein können (z. B. sorglos bei 9.41 Nachlässig und 10.38 Tollkühn).

Eine einfachere Formulierung der Aufgabenstellung bei der Einordnung führt dazu, dass neues Wortmaterial an den Stellen eingeordnet werden soll, an denen schon inhaltlich ähnliche Wörter vom gleichen grammatischen Typ stehen. Diese Aufgabenstellung lässt sich (zumindestens teilweise) automatisch lösen. Die für ein einzuordnendes Wort nötigen ähnlichen Wörter konnten auf zwei Wege ermittelt werden: Einmal verfügt das Wortschatz-Lexikon über Angaben zu Synonymen für rund 90 ooo Wörter. Zum anderen wurden die bereits in Abschnitt 2.1 erwähnten Kookkurrenzen benutzt: Ein Wort lässt sich wahrscheinlich dort sinnvoll einordnen, wo auch andere Wörter der gleichen Wortart stehen, die statistisch auffällig gemeinsam mit dem einzuordnenden Wort auftreten. Da für solche neu einzuordnenden Wörter eine Mindesthäufigkeit von 20 Vorkommen gefordert wurde, gab es in der Regel solche Kookkurrenzen.

Als nächstes wurden die neu aufzunehmenden Wörter in farbiger Schrift automatisch an den so ausgewählten Stellen in das Manuskript übernommen. Anschließend wurde das so erweiterte Manuskript von Hand durchgesehen und speziell die farbigen Bereiche begutachtet: Unpassende Vorschläge wurden entfernt, ggf. wurden Verschiebungen und Teilungen von semantischen Gruppen vorgenommen. Auch wenn hier ca. $50 \%$ der automatisch erzeugten Vorschläge zurückgewiesen wurden, beschränkte sich der manuelle Aufwand auf ein einmaliges Durcharbeiten des Manuskripts.

Dieser Bearbeitungsschritt hat die alphabetische Sortierung innerhalb der semantischen Gruppen zerstört. Diese wurde erst in einem allerletzten Bearbeitungsschritt des Manuskripts wieder hergestellt.

Nicht alle aufzunehmenden Wörter ließen sich so einordnen. Speziell bei Wörtern aus neuen Fachbereichen wie Computer, Sport oder Politik ergab sich in vielen Fällen

6 Sachgruppenbezogene Wörterbuchartikel haben in mehrerer Hinsicht andere Eigenschaften als Wörterbuchartikel in alphabetischen Wörterbüchern, worauf hier aber nicht eingegangen werden soll. 
keine Möglichkeit der automatischen Einordnung. Hier blieb nur die traditionelle Möglichkeit, die Wörter von Hand zusammenzufassen und neue Sachgruppen sowie semantische Gruppen einzurichten.

Die Konzeption der Neubearbeitung sah hier vor, die Kompetenz von externen Fachgutachtern einfließen zu lassen, um die fachliche Qualität zu sichern. Bei der vorliegenden achten Auflage gab es diese Fachbegutachtung für die Bereiche Literatur, Medizin, Musik, Politik, Sport und Technik.

Für die neunte Auflage bestand durch die unveränderte Sachgruppenstruktur ein fertiger Rahmen, der von automatischen Verfahren für die Einordnung aller neu aufzunehmenden Wörter genutzt werden konnte. Kandidaten für die Aufnahme in den Dornseiff waren vor allem Wörter unter den 150.000 häufigsten Wörtern in der deutschen Alltagssprache. Für diese Wörter gab es im Wortschatzkorpus mindestens 400 Belegstellen. Diese größeren Häufigkeiten kombiniert mit den in der Zwischenzeit neu entstandenen Verfahren wie sog. Word Embeddings ermöglichten für die neunte Auflage eine weitaus zielgenauere automatische Einordnung als bei der achten Auflage.

\subsection{Verweisungen auf andere sachgruppenbezogene Wörterbuchartikel}

Zum ersten Mal wurde in das Wörterverzeichnis ein Verweissystem nach systematischen Prinzipien integriert. Jeder sachgruppenbezogene Wörterbuchartikel hat einen Verweisteil, in dem Verweisangaben stehen, anhand derer der Benutzer Verweise erschließen kann, deren Befolgung ihn zu einem anderen Wörterbuchartikel führt.

Die semantische Verwandtschaft, die die wörterbuchgegenstandsbedingte Verweisvoraussetzung bildet, kann verschiedener Art sein, wie die folgenden Beispiele zeigen:

- Gegensatz: von 2.13 Mann auf 2.14 Frau

- Über- oder Unterordnung: von 20.47 Autoindustrie auf 20.46 Industrie

- Unterbegriffe zum gleichen Oberbegriff: von 8.14 Schiff auf 8.15 Flugzeug

- Teil-Ganzes-Beziehung: von 22.8 Teufel auf 22.20 Hölle

- Typische Eigenschaften: von 8.15 Flugzeug auf 8.19 Schnell

- Ursache und Wirkung: von 5.47 Unglück auf 20.17 Verlust

- andere Aspekte des Themas: von 10.37 Mut auf 5.35 Kraft

Sind zwei Wörterbuchartikel durch solch eine semantische Verwandtschaft verbunden, so enthalten sie in der Regel mehrere Wörter oder Wortgruppen gemeinsam. Damit bot sich eine Möglichkeit, mit automatischen Mitteln Vorschläge für neue Verweisangaben zu erzeugen: Enthalten nämlich zwei Wörterbuchartikel mehrere Wörter oder Wortgruppen gemeinsam, sind möglicherweise Verweise zwischen diesen Artikeln sinnvoll. Als angebracht erwies sich eine Minimalzahl von vier gemeinsamen Wörtern oder Wortgruppen, um einen Verweisvorschlag zu erzeugen. Auch dies musste, zusammen 
mit der Liste bisheriger Verweise, natürlich wieder manuell überprüft werden, wobei etwa die Hälfte der Vorschläge verworfen wurde. Außerdem wurden einige zusätzliche Verweise von Hand eingefügt. Insgesamt sind jetzt reichlich 6100 Verweise enthalten.

\subsection{Inhaltliche Änderungen}

In der siebten Auflage sind die einzelnen sachgruppenbezogenen Wörterbuchartikel im Durchschnitt etwa eine halbe Seite lang. Es gibt zwei auffällige Ausnahmen: Die Artikel 2.2 Pflanzenarten und 2.9 Tierarten mit 81 bzw. 34 Seiten. Ab der achten Auflage enthalten diese beiden Wörterbuchartikel auch nur noch die allgemein bekannten Bezeichnungen der Pflanzen und Tiere; verzichtet wurde auf Vollständigkeit, lateinische Bezeichnungen sowie den Versuch, alle regionalen Bezeichnungen zu erfassen.

Die Menge der aufgenommenen Eigennamen wurde reduziert: Weitgehend verzichtet wurde auf die in der siebten Auflage vorhandenen Personen aus Mythologie und Literatur, da einerseits die in der siebten Auflage vorhandene Auswahl unsystematisch und unvollständig erscheint, andererseits mit der gewählten korpusbasierten Methode keine Verbesserung zu erreichen war. Beibehalten wurden jedoch Eigennamen in den Artikeln 2.2 Pflanzenarten, 2.8. Tierarten, 13.20 chemische Elemente und 15.1 Vornamen. Auch hier wurde korpusbasiert ausgewählt, so dass Bekanntheit und nicht Vollständigkeit angestrebt wurden.

\section{Die sachgruppenbezogenen Wörterbuchartikel und ihre Anordnung}

\subsection{Grobgliederung nach Sachgruppen}

Anliegen des Wörterbuches ist es nach wie vor, die ausgewählten Wörter und Wortgruppen der deutschen Alltagssprache (in ihrer Verzahnung mit anderen Sprachvarietäten) nach Sachgruppen begrifflich zu ordnen. Da wir Wörter benutzen, um über die Dinge der Welt, über Vorgänge und ihre Zusammenhänge zu reden, ist mit der Ordnung der Sachgruppen auch eine Sicht auf die Welt gegeben. Eine solche Anordnung muss einigen naheliegenden Kriterien gehorchen. So sollte nahe beieinander stehen, was unter Anwendung der gewählten Methoden als zusammengehörig gelten kann. Die Umsetzung der komplexen semantischen Zusammenhänge zwischen den Bezeichnungen der Sachgruppen in eine lineare Abfolge ist immer problematisch und wird auch die subjektive Sicht des Autors wiedergeben. Im Zeitalter von Internet und Hypertext verliert allerdings die im gedruckten Buch notwendige lineare Abfolge ihre zentrale Bedeutung und wird ersetzt durch die Forderung nach einer sinnvollen und nachvollziehbaren Vernetzung der einzelnen Sachgruppen. In diesem Sinne schafft die elek- 
tronische Version einer solchen Sammlung erstmals die angemessenen Orientierungsmöglichkeiten.

Der Deutsche Wortschatz nach Sachgruppen ist nach 970 Sachgruppen gegliedert, die zu 22 Hauptgruppen zusammengefasst sind. Diese Einteilung folgt im Prinzip den Ideen von Franz Dornseiff und seiner Gliederung der siebten Auflage. Wesentlich erweitert wurden die Bereiche Politik, Wirtschaft, Technik, Recht, Medizin und Sport. Weiter wurden Termini aus verschiedenen Wissenschaften aufgenommen, so dass Wissenschaften erstmals als Sachgruppen auftreten.

Die folgende Tabelle vergleicht die Übersicht über die Hauptgruppen der siebten mit den folgenden Auflagen.

\section{Auflage}

Anorganische Welt. Stoffe

2 Pflanze. Tier. Mensch (Körperliches)

3 Raum, Lage, Form

Größe, Menge, Zahl, Grad

Wesen, Beziehung, Geschehnis

6 Zeit

7 Sichtbarkeit, Licht, Farbe, Schall, Temperatur, Gewicht, Aggregatzustände, Geruch, Geschmack

8 Ortsveränderung

Wollen und Handeln

Sinnesempfindungen

11 Fühlen, Affekte, Charakter-eigenschaften

12 Das Denken

13 Zeichen, Mitteilung, Sprache

14 Schrifttum, Wissenschaft

15 Kunst und Kultur

16 Gesellschaft und Gemeinschaft

17 Geräte, Technik

18 Wirtschaft

19 Recht, Ethik

20 Religion, Übersinnliches

\section{8. und 9. Auflage}

Natur und Umwelt

Leben

3 Raum, Lage, Form

4 Größe, Menge, Zahl, Grad

5 Wesen, Beziehung, Geschehnis

6 Zeit

7 Sichtbarkeit, Licht, Farbe, Schall, Temperatur, Gewicht, Aggregatzustände

jetzt in 16

8 Ort, Ortsveränderung

9 Wollen und Handeln

0 Fühlen, Affekte, Charaktereigenschaften

11 Das Denken

12 Zeichen, Mitteilung, Sprache

13 Wissenschaft

14 Kunst und Kultur

15 Menschliches Zusammenleben

16 Essen und Trinken

17 Sport und Freizeit

18 Gesellschaft

19 Geräte, Technik

20 Wirtschaft, Finanzen

21 Recht, Ethik

22 Religion, Übersinnliches

\section{Bemerkung}

Titel geändert

Titel geändert

jetzt in 7 und 16

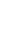

Schrifttum in 13

16 (alt) aufgeteilt

in 15 und 18 


\subsection{Feingliederung der sachgruppenbezogenen Wörterbuchartikel}

Die sachgruppenbezogenen Wörterbuchartikel enthalten Wörter und Wortgruppen, welche in verschiedenen Relationen zum Sachgruppentitel stehen können. Die so entstehenden semantischen Gruppen sind jeweils durch fette quadratische Trennzeichen voneinander getrennt, die Wörter oder Wortgruppen innerhalb einer solchen semantischen Gruppe sind durch kleine Mittenpunkte getrennt. Jede semantische Gruppe enthält nur Wörter einer Wortart. Die Ordnung innerhalb einer Gruppe ist alphabetisch, wobei zur besseren Orientierung jeder Gruppe ein für diesen Zweck als geeignet geltender Ausdruck (außerhalb der alphabetischen Reihenfolge) vorangestellt sein kann. Ab der 9. Auflage sind diese Wörter fett hervorgehoben. Da der Dornseiff einen Benutzer voraussetzt, der die deutsche Sprache gut beherrscht und dem es nur darum geht, nicht verfügbare Wörter aufzufinden, wurde innerhalb der semantischen Gruppen eine asemantische alphabetische Ordnung bevorzugt, damit der Benutzer bei der Wahl seines Ausdrucks nicht beeinflusst wird, sondern auf Grund seiner semantischen Kompetenz relativ zur jeweiligen Intention selbständig entscheiden kann. Außerdem unterstützt die alphabetische Ordnung indirekt die separierende Funktion des fetten quadratischen Trennzeichens.

Nur in wenigen Fällen wurde von dieser alphabetischen Reihenfolge abgewichen, nämlich in den Fällen, in denen die aufgezählten Wörter eine andere, allgemein anerkannte historisch-pragmatisch eingespielte Reihenfolge haben. Beispiele sind die zeitliche Abfolge bei Jahreszeiten, die Ordnung nach Größe bei Maßeinheiten oder die Ordnung der chemischen Elemente nach dem Periodensystem.

Jeder Wörterbuchartikel besteht aus einem Titel, einem Verweisteil und einem Formenteil. Der Verweisteil enthält Verweisangaben; diese ermöglichen den Zugriff auf andere Artikel, die in einem engen inhaltlichen Zusammenhang mit dem Ausgangsartikel stehen. Mit der Verweisangabe wird der vollständige Sachgruppentitel als Verweisadresse genannt, damit der Leser sofort entscheiden kann, ob er dem Verweis folgen möchte oder nicht. Die Reihenfolge der Verweisangaben richtet sich nach den Nummern in den Sachgruppentiteln.

Der Formenteil enthält Angaben von Wörtern und Wortgruppen. Diese sind zunächst nach Wortarten sortiert und erscheinen in der Reihenfolge Substantive - Adjektive / Adverbien - Verben. Ihrer Struktur nach sind sie nach Wörtern und Wortgruppen getrennt.

Weiterhin sind bei einigen Sachgruppen am Anfang des Formenteils Affixe, Affixoide oder gelegentlich Bestandteile von Komposita angegeben, und zwar solche, die geeignet sind, die in dem Artikel genannten Wörter durch Wortbildung zu ergänzen.

Am Anfang des Formenteils (ggf. nach den Affixen) werden außerdem Ausrufe angeführt. 


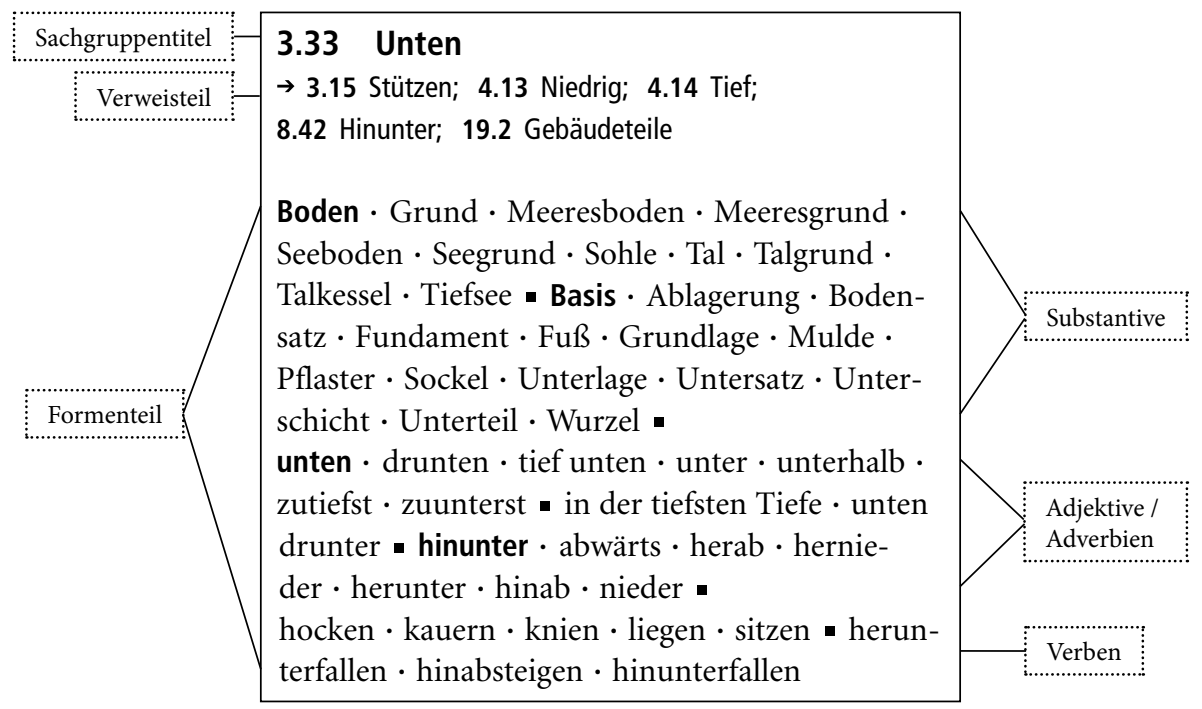

Im Rahmen einer Wortart erfolgt eine Trennung in semantische Gruppen. Verschiedene semantische Relationen lassen sich zwischen dem Sachgruppentitel und den semantischen Gruppen identifizieren, aber auch innerhalb einer semantischen Gruppe und über die semantischen Gruppen hinweg.

Ein sachgruppenbezogener Wörterbuchartikel lässt sich als Ausschnitt aus einem semantischen Netz interpretieren, bei dem die semantischen Gruppen in einer bestimmten semantischen Relation zum Sachgruppentitel stehen.

Solche Relationen in den semantischen Gruppen von Substantiven können sein:

- Wörter ähnlicher Bedeutung (d.h. Synonyme in einem weiteren Sinne); z. B. in 10.16 Wohlgefallen, Bewundern, Schönheit: Anmut · Blüte $\cdot$ Charme $\cdot$ Ebenmaß $\cdot$ Eleganz · Grazie $\cdot[\ldots]$

- Oberbegriff - Unterbegriff (oder, anders ausgedrückt Hyperonymie bzw. Hyponymie); z. B. in 14.10 Film und Kino: Film · Abenteuerfilm · Actionfilm · Actionkomödie $\cdot$ Actionthriller $\cdot$ Animationsfilm $\cdot$ Antikriegsfilm $\cdot[\ldots]$

- Teil-Ganzes-Beziehung (Partonymie); z. B. in 12.58 Buch, Heft: Abschnitt · Anführung $\cdot$ Artikel $\cdot$ Bibliografie $\cdot$ Buchillustration $\cdot$ Kapitel $\cdot$ Nachwort $\cdot[\ldots]$

- Hilfsmittel und Werkzeuge (Instrument); z. B. in 9.12 Veranlassung, Beweggrund: Blickfang $\cdot$ Köder $\cdot$ Lockmittel $\cdot$ Magnet $\cdot$ süße Worte $\cdot$ verbotene Frucht

- Assoziierte Ausdrücke aus dem dazugehörigen Wortfeld, z. B. Träger einer Eigenschaft in 16.17 Wohlgeschmack: Delikatesse · Feinkost - Leckerbissen · Leckerei

- Ortsangaben; z. B. in 2.28 Geburt, Abtreibung: Abtreibungsklinik - Babyklappe · Brutkasten · Entbindungsstation · Frauenabteilung $\cdot[\ldots]$

- Zeitangaben; z.B. bei 17.28 Reise, Tourismus, Urlaub: Ankunftszeit · Ferienbeginn · Ferienmonat $\cdot$ Ferienwoche $\cdot$ Hauptreisezeit $\cdot$ Reiseantritt $\cdot[\ldots]$ 
- Maßangaben; z.B. 19.16 Computer: Byte $\cdot$ Kilobyte $\cdot$ Megabyte $\cdot$ Gigabyte

- Tiere; z. B. in 8.5 Beförderung: Brieftaube $\cdot$ Esel $\cdot$ Kamel $\cdot$ Packesel $\cdot$ Pferd

- Personenbezeichnungen, unterteilt in Gruppen und Einzelpersonen; z. B. in 8.9 Straße: Verkehrssünder · Alkoholsünder · Autoknacker · Falschfahrer · Falschparker - Geisterfahrer · [...] und in 11.20 Ansicht: Glaubensgemeinschaft · Kirche · Partei · Schule $\cdot$ Sekte

Neben den analogen Relationen in den semantischen Gruppen von Adjektiven / Adverbien und Verben, die jeweils durch die Zuordnung der jeweiligen Wörter zu den semantischen Gruppen der Substantive entstehen, gibt es folgende weitere Unterscheidungen:

- Verben nach reflexiv und transitiv. In 4.52 Geringerer Grad finden sich zwei Gruppen zum Verb verschlechtern, nämlich: sich verschlechtern · sinken · zurückbleiben etw. verschlechtern · senken · unterbieten · zügeln.

- Verben zur Beschreibung von Vorgängen bzw. von Zuständen; z. B. in 11.20 Ansicht glauben · annehmen · sich ausmalen · beharren · denken · [...] . bekehren . beruhigen $\cdot$ einreden $\cdot$ lehren $\cdot \ldots$

Innerhalb einer semantischen Gruppe finden sich häufig wortfeldintern assoziierte Ausdrücke, beispielsweise in 21.31 Polizei, Vollziehungsbeamte: [...] Polizeikommissariat . Polizeipräsidium $\cdot$ Polizeiverwaltung $\cdot$ Polizeizentrale [...].

Über die semantischen Gruppen hinweg findet man dagegen oft wortfamilienintern assoziierte Ausdrücke. Wo dies sinnvoll erschien, wurden diese auch als erste Einträge in den semantischen Gruppen gewählt, wie bei 2.31 Erholung: Erholung [...] • erholt [...] • erholsam [...] • erholen [...]. 